\title{
Light-modulated cationic and anionic transport across protein biopolymers
}

\author{
Alex Burnstine-Townley, Somen Mondal, Yuval Agam, Ramesh Nandi, and Nadav Amdursky* \\ Schulich Faculty of Chemistry, Technion - Israel Institute of Technology, Haifa 3200003, Israel. \\ *Corresponding Author: Nadav Amdursky, E-mail: amdursky@technion.ac.il
}

\begin{abstract}
Light is a convenient source of energy and the heart of light-harvesting natural systems and devices. Here, we show light-modulation of both the chemical nature and ionic charge carrier concentration within a protein-based biopolymer that was covalently functionalized with photoacids or photobases. Using steady-state and time-resolved fluorescence, we explore the capability of the biopolymer-tethered photoacids and photobases to undergo excited-state proton transfer and capture (ESPT and ESPC), respectively. Various electrical measurements show that both the photoacid- and photobase-functionalized biopolymers exhibit an impressive increase in ionic conductivity upon light irradiation, which can be modulated by the light intensity. Whereas ESPT-induced cationic protons are the charge carriers for the photoacid-functionalized biopolymer, ESPC-induced water-derived anionic hydroxides are the suggested charge carriers for the photobase-functionalized biopolymer. Our work introduces a versatile toolbox to lightmodulate charge carriers in polymers and taking together the attractive environmental nature of our new light-modulated ionic-conductive biopolymers, they can be considered for various photoelectrochemical applications.
\end{abstract}

\section{Keywords}

proton exchange membranes, anion exchange membranes, biopolymers, excited-state proton transfer, photoacids, photobases 


\section{Introduction}

Ionic conducting polymers are gaining high momentum over the last decade, and especially for energy-related applications, such as fuel cells, flow batteries, and capacitors (e.g., recent reviews ${ }^{[1-}$ ${ }^{6]}$ ). In general, the ionic charge carriers can be divided to positive cations and negative anions, wherein protons and hydroxide are the most common and targeted examples, respectively. These polymers are referred to as proton exchange membranes (PEM) and anion exchange membranes (AEM). In terms of the ionic transport mechanism, protons are transferred via water molecules and protonable oxo-acids (such as carboxylic or sulfonic acids), while hydroxides are transferred via water molecules and cationic functional groups (such as ammonium or imidazolium). ${ }^{[7]}$

Recently, new light-modulated capabilities have been introduced to control the protonic conductivity of $\mathrm{PEM},{ }^{[8-12]}$ as well as of non-polymeric proton-conducting metal-organic frameworks, ${ }^{[13-17]}$ crystals, ${ }^{[18-19]}$ and graphene oxide materials, ${ }^{[20]}$ targeting applications of photoelectric conversion and photoelectrodialysis. In all these examples, the light-modulated functionality was introduced to the proton conducting material using a photoacid, a molecule that releases a proton upon light irradiation. Two different types of photoacids have been used. The first are Brønsted-Lowry photoacids, exhibiting a major change in their acidity properties $\left(\mathrm{p} K_{\mathrm{a}}\right)$ upon light irradiation, and accordingly, undergo excited-state proton transfer (ESPT) to afford dissociation. The most notable example for such photoacid, which was also used in all of the studies that used a Brønsted-Lowry photoacid, ${ }^{[8,10-12,18,20]}$ as well as in our work here, is pyranine (8-hydroxypyrene-1,3,6-trisulfonic acid, HPTS) with ground state $\mathrm{p} K_{\mathrm{a}}$ of $\sim 7.4$ and excited state $\mathrm{p} K_{\mathrm{a}}{ }^{*}$ of $\sim 0.3-1.3$. Importantly, the ESPT process is fast with timescales of proton dissociation in the order of 100 ps for HPTS in water, which can be faster for super-photoacids. ${ }^{[21-22]}$ This type of photoacid was also used to light-trigger several $\mathrm{pH}$-responsive dynamic systems. ${ }^{[23-24]}$ The second type of photoacids (or better to say photoacid generators) are molecules undergoing photoisomerization, meaning that upon light irradiation they change their chemical structure leading to a proton release. The most notable example for such photoacid, which was also used in all of the literature studies that used a photoisomerized photoacid, ${ }^{[9,13,15,19]}$ is the spiropyranmerocyanine system. Due to the molecular isomerization, this process is much slower compared to ESPT.

We also introduce here the use of a photobase to light-modulate the ionic conductive properties of our material. We will use here a Brønsted-Lowry photobase, meaning that the molecule will undergo an excited-state proton capture (ESPC) and protonate following light absorption due to the much larger excited $\mathrm{p} K_{\mathrm{a}}{ }^{*}$ compared to the ground-state $\mathrm{p} K_{\mathrm{a}}$. Like Brønsted-Lowry photoacid, the ESPC process of this photobase is fast. Unlike the mentioned use of photoacids in manipulating ionic properties, the use of Brønsted-Lowry photobases for the conjugate purpose was never reported. Another class of photobases is an Arrhenius type, resulting in a hydroxide release following light absorption, which was recently used to manipulate ionic properties. ${ }^{[20]}$ However, the reversibility of the latter class is poor and the return of the hydroxide in the ground state is very slow with very poor efficiency, ${ }^{[23]}$ which is in contrast to Brønsted-Lowry photo-acids/bases. 
A far throw from common ionic conductive materials, nature also uses the controlled movement of ions in numerous fundamental biochemical processes. In some cases, this control is lightinitiated, such as in the photosystem, in which light irradiation induces the start of an electron transport relay that in turn induces the translocation of protons across the membrane, and the opsin family, a light-triggered ion pump, which is used nowadays in optogenetics applications. In all cases, the directional ion transport molecule is a protein. Accordingly, in recent years we witness the emergent use of proteins as the building blocks for the formation of ionic conducting

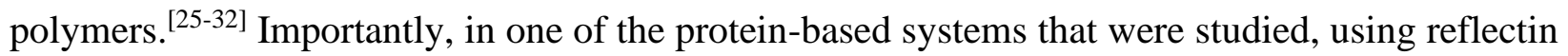
films, there was an attempt to use the HPTS photoacid to light-modulate the protonic conductivity, ${ }^{[11]}$ however the observed magnitude of the response was rather low, of only a few percentages. We speculate that it was due to the mixing of the photoacid in low concentration into the film and not covalently attach it to the surface of the protein, especially as it is known that the protein surface has a fundamental role in dictating protonic transport. ${ }^{[29-30,33]}$

In here, we work with the bovine serum albumin (BSA) protein as the starting material for the polymer, which can form free-standing insoluble polymers. ${ }^{[29,34-35]}$ An added value of choosing BSA is its sustainable nature, being one of the waste products of the bovine industry, resulting in its large availability and low price. We use here the BSA to form electrospun mats (a detailed analysis of the mats microstructure can be found in our recent work $\left.{ }^{[33]}\right)$, followed by covalent binding of either a photoacid or a photobase to the protein functional groups. We follow both the steady-state and time-resolved fluorescence properties of the polymers as well as the electrical properties of it to explore the dynamicity of the ESPT and ESPC and their influence on the electrical properties while discussing different modes of ionic transport.

\section{Results and Discussion}

\section{Photoacid-modified protein biopolymer}

At first, we will show the ability to light-modulate protons as charge carriers within BSA electrospun mats. We hypothesize that covalent binding of the photoacid to the surface of the protein biopolymer will result in a significant light-modulation of the protonic transport. While our chosen HPTS photoacid is not the strongest one, it absorbs in the visible ( 400 nm), enabling the use of common and biologically safe visible light sources. To attach the HPTS to the BSA mat, the HPTS was modified to a triply electrophilic sulfonyl chloride derivative (HPTS-Cl) (Figure 1aFigur). The electrophilic sulfonyl chlorides receive nucleophilic attack from amine and hydroxy groups of the protein amino acids residues, resulting in sulfonamides and sulfonate esters, respectively. One to four reagent equivalents of the reactant, relative to the nucleophiles present in BSA, were added to the mat to explore the effect of increasing concentration of the tethered photoacid (further details in the Methods section).

Our working principle is that upon light irradiation the tethered photoacid will undergo ESPT, resulting in a proton dissociation from the photoacid (Figure 1b). Following ESPT, the proton can 
be accepted by several functional groups. The straightforward proton acceptors are water molecules, followed by the formation of hydronium, while other proton acceptors are the protein functional groups. In both cases, we foresee that the light-modulated release of a proton from the photoacid will result in an increase of the cationic protonic charge carrier concentration within the BSA mat. On the other hand, there is a competitive process in the form of proton geminate recombination; the rapid recombination of the proton with the excited deprotonated photoacid. This process will prevent delivering the proton (as a charge carrier) to the hydrogen bond network of the BSA mat comprising water molecules and protein functional groups. Upon turning the light off, the deprotonated HPTS in its ground state $\left(\mathrm{RO}^{-}\right)$acts as a strong base and will protonate, resulting in a decrease in cationic charge carriers and a reduction of the proton transport efficiency across the mat to the original value.

(a)

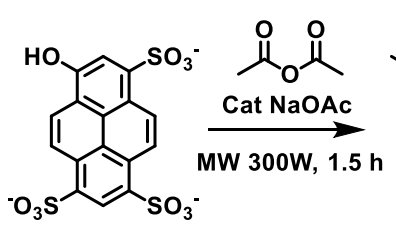

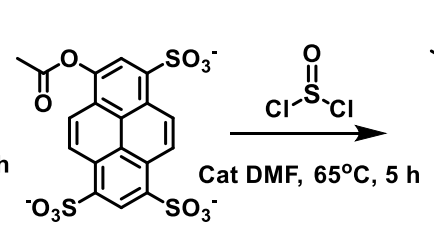

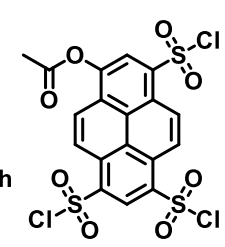

$\mathrm{Cl}_{\mathrm{O}}$
BSA Mat

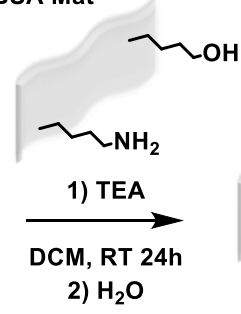

(b)

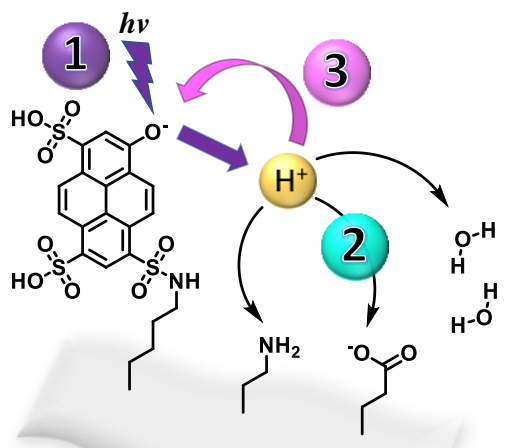

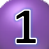

Light-induced change in pKa resulting in proton dissociation

2 water moler functional groups in the BSAmat

\section{3}

Proton recombination

Figure 1. Photoacid-modified BSA mat. (a) Scheme of HPTS modification and conjugation to the BSA mat. (b) Schematic of the light-induced processes within the HPTS-modified BSA mat.

An important validation step is to investigate the ESPT efficiency and the proton recombination process. As shown in different studies using the HPTS at various biological environments, such as within protein (and even with the BSA protein and mat), $\left.{ }^{[2,}, 36-40\right]$ membranes ${ }^{[41]}$ or polysaccharides, ${ }^{[38,42]}$ the ESPT process of HPTS varies dramatically, all the way to a very poor ESPT at more hydrophobic pockets. Recently, it was also shown that the excited-state properties of HPTS on the surface of different polymers can vary dramatically. ${ }^{[43]}$ To explore the ESPT of the bound HPTS, we turn to fluorescence measurements; since the protonated and deprotonated species of the photoacid emit at different wavelengths, it is straightforward to follow them 
separately as a function of time. Following light excitation and the ESPT process, we should mainly observe the $\mathrm{RO}^{-*}$ form: $\mathrm{ROH} \stackrel{h v}{\longrightarrow} \mathrm{ROH}^{*} \underset{\text { Rec. }}{\stackrel{E S P T}{\longrightarrow}} \mathrm{RO}^{-*}+\mathrm{H}^{+}$, whereas excited-state recombination results in the re-formation of the protonated $\mathrm{ROH}^{*}$, which is an unwanted process for us (Figure 1b). As can be observed in the steady-state emission spectrum of the HPTS-modified BSA mat (Figure 2a), the $\mathrm{RO}^{-*}$ is the only peak, which already implies the high efficiency of the ESPT process. Interestingly, the presence of only the $\mathrm{RO}^{-*}$ peak is rather similar to the spectrum of solvated HPTS, yet it is in contrast to the spectrum of HPTS that was bound to a binding site within BSA (Figure S1 and text within). ${ }^{[36,40]}$ The latter highlights the fundamental difference of the excited-state properties of HPTS between being covalently bound to the BSA surface compared to being physisorbed into a binding site of the BSA protein. To determine the ESPT efficiency, i.e., its rate constant, as well as the possible proton geminate recombination in the excited-state we turned to time-resolved measurements (Figure $2 \mathrm{~b}$ ). The decay of the $\mathrm{ROH}^{*}$ form exhibits an initial very fast decay with a lifetime of $90 \mathrm{ps}\left(k_{E S P T}=1.1 \times 10^{10} \mathrm{~s}^{-1}\right)$, corresponding to a very fast ESPT process. Due to this very fast ESPT, the $\mathrm{ROH}^{*}$ peak is absent in the time-integrated measurement, i.e., the steady-state spectrum. However, upon reconstructing the time-resolved emission spectrum (TRES), we can observe the presence of the $\mathrm{ROH}^{*}$ peak at short timescales of $0.5 \mathrm{~ns}$ (Figure 2a inset). Surprisingly, the ESPT efficiency of the HPTS-tethered BSA mat is even slightly more efficient than the one of HPTS solvated in water, and the main difference is the different recombination efficiency within the BSA mat, manifested as a long-lived component at longer time scales, which is probably due to the restricted dimensionality within the BSA mat compared to bulk water (Figure S1 and text within). In accordance with the steady-state measurements, the excited-state dynamics of the HPTS-tethered BSA mat are fundamentally different than those of HPTS within the binding site of BSA, ${ }^{[36,40]}$ the latter showing much slower ESPT efficiency as well as higher recombination efficiency. Figure $2 b$ also shows the transient of the $\mathrm{RO}^{-*}$ peak that rises time in accordance with the fast ESPT of the $\mathrm{ROH}^{*}$ decay (inset). In a short conclusion, in this important step, we validated the ability of the HPTS photoacid to donate a proton upon light irradiation with high efficiency, and the stronger the light intensity the more protons are being released, whereas according to our hypothesis (Figure 1b) it should induce an increase in protonic charge carriers. 

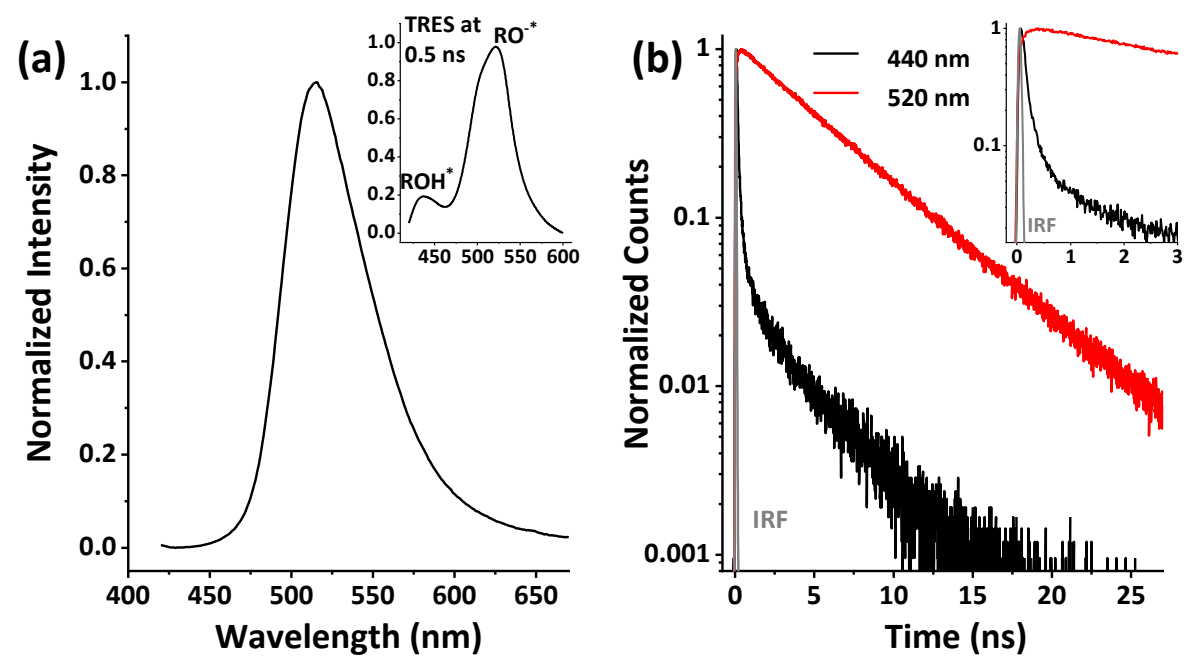

Figure 2. Excited-state dynamics of photoacid-modified BSA mat. (a) Steady-state and (b) time-resolved fluorescence of the HPTS-tethered BSA mat, with $\lambda_{\text {ex }}=405 \mathrm{~nm}$. The inset in (a) is the TRES taken at $0.5 \mathrm{~ns}$. The inset in (b) is a zoom-in of the first $3 \mathrm{~ns}$, emphasizing the fast decay of the $\mathrm{ROH}^{*}$ (at $440 \mathrm{~nm}$ ) and the rise time of the $\mathrm{RO}^{-*}$ (at $520 \mathrm{~nm}$ ). Panel (b) also shows the instrument response function (IRF).

In our exploration of the light-modulated change in the electronic properties of the HPTS-tethered BSA mat, we used impedance analysis, where a frequency-dependent AC bias is applied across the material while measuring the impedance response. The main advantage of this technique, and especially for measuring ionic transport is its ability to decouple between bulk resistance, manifested at high frequencies, to other processes taking place at the contact, manifested at much lower frequencies. We showed previously that the native BSA mat already exhibits some protonic conductivity, in the order of $0.1 \mathrm{mS} \cdot \mathrm{cm}^{-1} \cdot{ }^{[30,44]}$ Interestingly, following HPTS conjugation to the BSA mat, we already observed an increase in the mat conductivity (Figure $3 \mathrm{a}$ and $3 \mathrm{~b}$ ). This increase can be ascribed to an increase in the concentration of oxo-acids, namely the sulfonyls of HPTS, resulting in an increase in the H-bonds network population required for proton transport (further discussion below). However, and surprisingly, while going from 1 to 4 equivalents of tethered HPTS we have found an inverse relation between the concentration of HPTS within the BSA mat to the measured protonic conductivity, whereas the more HPTS tethered to the BSA mat the less increase in conductivity in comparison to the native mat (Figure $3 b$ and Table 1). This peculiar finding might be a result of the plural nucleophilic attack on HPTS-Cl, creating uncharged sulfonyl derivates rather than sulfonates. In terms of the light-modulation response of the measured conductance (Figure 3a and 3b), the larger the concentration of HPTS within the mat, the larger fold increase in the measured protonic conductivity upon light irradiation. As such, while using 4 equivalents of HPTS we reached a remarkable $5.4 \pm 1.3$ folds increase in conductivity following light irradiation (at $405 \mathrm{~nm}$ ), bringing the conductivity from $0.13 \pm 0.04 \mathrm{mS} \cdot \mathrm{cm}^{-1}$ in dark to $0.71 \pm$ $0.18 \mathrm{mS} \cdot \mathrm{cm}^{-1}$ under light irradiation (Table 1). Next, we turned to using light as a gating source to control the protonic charge carriers within the HPTS modified BSA mat. As shown in Figure 3c, 
the measured impedance response of our system is highly tunable in accordance with the power intensity of the light.
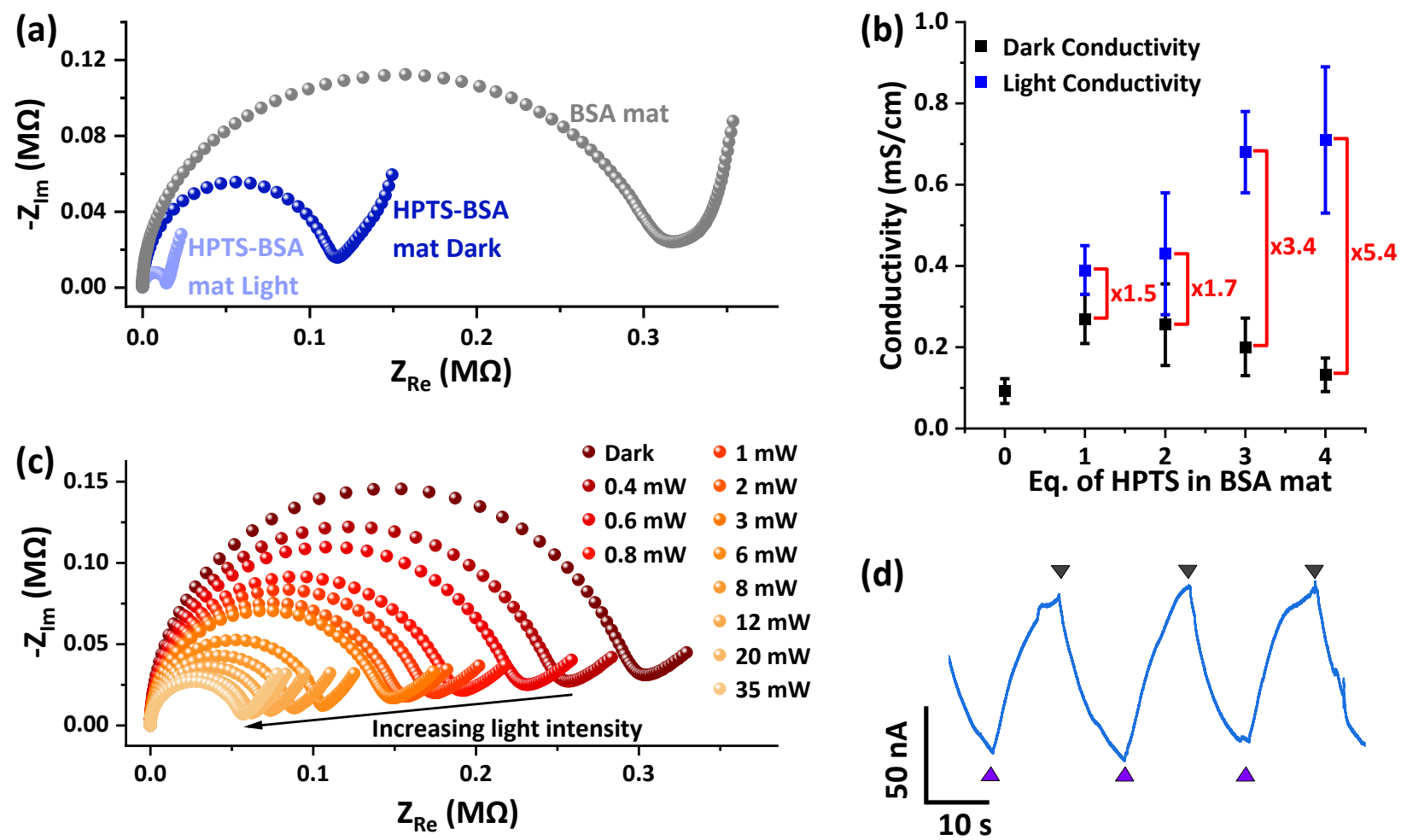

Figure 3. Light-modulated electrical response of photoacid-modified BSA mat. (a) Impedance measurements of the BSA mat compared to the 4 equivalents HPTS-tethered BSA mat at dark and under illumination. (b) A summary of the extracted conductivities in dark and light. (c) Light modulation of the impedance for the 4 equivalents HPTS-tethered BSA mat as a function of light intensity. (d) Current-time measurements (at $0.5 \mathrm{~V}$ ) of the 4 equivalents HPTS-tethered BSA mats upon intervals of light irradiation (marked with triangles).

Table 1. Summary of extracted conductivities $(\sigma)$ from the HPTS- and 9AA-tethered BSA mats.

\begin{tabular}{|c|c|c|c|}
\hline & $\begin{array}{l}\text { Measured at dark } \\
{\left[\times 10^{-4} \mathrm{~S} \cdot \mathrm{cm}^{-1}\right]}\end{array}$ & $\begin{array}{l}\text { Upon light irradiation }^{\mathrm{a}} \\
\left.\qquad \times 10^{-4} \mathrm{~S} \cdot \mathrm{cm}^{-1}\right]\end{array}$ \\
\hline \multicolumn{2}{|c|}{ Native BSA mat } & \multicolumn{2}{|c|}{$1.0 \pm 0.2$} \\
\hline \multirow{4}{*}{ 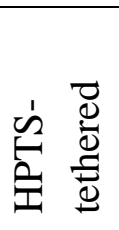 } & 1 eq. & $2.7 \pm 0.6$ & $3.9 \pm 0.6$ \\
\hline & 2 eq. & $2.6 \pm 1.0$ & $4.3 \pm 1.5$ \\
\hline & 3 eq. & $2.0 \pm 0.7$ & $6.8 \pm 1.0$ \\
\hline & 4 eq. & $1.3 \pm 0.4$ & $7.1 \pm 1.8$ \\
\hline \multirow{4}{*}{ 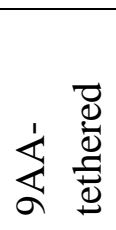 } & 1 eq. & $0.57 \pm 0.05$ & $0.71 \pm 0.02$ \\
\hline & 2 eq. & $0.55 \pm 0.06$ & $0.79 \pm 0.15$ \\
\hline & $3 \mathrm{eq}$. & $0.45 \pm 0.06$ & $1.02 \pm 0.08$ \\
\hline & 4 eq. & $0.39 \pm 0.05$ & $1.38 \pm 0.14$ \\
\hline
\end{tabular}

${ }^{\mathrm{a} E x c i t e d}$ at $405 \mathrm{~nm}$. 
In addition to our impedance analysis, we performed also current-time (I-T) measurements upon the application of a DC bias. The main problem in using gold electrodes (as in our study) for measuring ionic transport is the ion-blocking nature of such electrodes, meaning that upon the application of a DC-bias, ions will quickly accumulate on the surface of the electrode, resulting in a significant reduction of the measured current, and eventually its saturation. Accordingly, ionic conductivity is measured using frequency-dependent impedance measurements (vide supra). An exception for this is protons, in which gold electrodes were proven to be suitable for measuring protonic current even upon application of a DC-bias (in a yet-to-be-discovered electrochemical process). ${ }^{[29-30,45]}$ However, in contrast to the impedance measurements, transient current measurements allow deciphering the time-responsivity of our system following light irradiation. As shown in Figure 3d, the transient I-T measurement indicates that the time-response of our HPTS-BSA mat system is on the second's timescale and highly reversible. As discussed, one of the differences between Brønsted-Lowry photoacids to photoacids generators is the different timeresponse of the molecular system. Indeed, the time-responsiveness of our system (seconds) is faster than the one of photoacids generators (minutes). ${ }^{[13,15,19]}$

The last electrical measurement of the HPTS-modified BSA mats is based on a protonic fieldeffect transistor (FET). In such a device, a DC bias is applied in-plane across the BSA mat (between the source and drain electrodes), while a third gating electrode is situated beneath the BSA mat separated by an insulating oxide layer. FET measurements are highly informative as they allow the direct calculation of the charge carrier (protons) mobility $(\mu)$ across the material while measuring the change in source-drain current $\left(I_{D S}\right)$ relative to a change in gate voltage $\left(V_{G S}\right)$ :

$$
\mu= \pm \frac{\partial I_{D S}}{\partial V_{G S}} \cdot \frac{L}{W C_{G S} V_{D S}}
$$

where $C_{G S}$ is the capacitance of the oxide layer, $V_{D S}$ is the source-drain voltage and $W$ and $L$ is transistor area width and length, respectively. Importantly (and especially to our next part of the study), due to the ability of using gold electrodes as proton-transparent electrodes, protonic FET using gold electrodes is doable, while other ionic FET cannot be achieved using gold electrodes. We have used the 4 equivalents HPTS-modified BSA mat for our FET measurements as it shows the largest light-modulation capability (Figures $3 \mathrm{~b}$ ). The protonic conductivity of this HPTS-BSA mat (in the dark) is $\sim 30 \%$ larger than the unmodified native BSA mat (Table 1). While using Eq. (1) to the FET measurements of this HPTS-mat in the dark (Figure 4, dashed lines), we extracted a protonic mobility value of $5 \times 10^{-3} \mathrm{~cm}^{2} \mathrm{~V}^{-1} \mathrm{~S}^{-1}$, which is $\sim 25 \%$ larger compared to the mobility measured for the native BSA mat $\left(\sim 4 \times 10^{-3} \mathrm{~cm}^{2} \mathrm{~V}^{-1} \mathrm{~S}^{-1}\right)$. Considering the Nernst-Einstein relation: $\sigma=\mu n e$, we can conclude that the measured increase in the conductivity $(\sigma)$ of the HPTSmodified BSA mat in the dark is solely due to the increase in charge mobility, and not due to an increase in charge carrier concentration $(n)$. This new finding highly supports our notion for the observed increase of protonic conductance upon modifying the mat with HPTS. As stated, the addition of HPTS introduces sulfonic acids to the mat (Figure 1). These sulfonic acids can integrate 
into the existing H-bonds network and improve it, and by doing so they increase the mobility of the protonic charge carriers in a Grotthuss-like transport mechanism, ${ }^{[46]}$ meaning they provide more proton hopping sites and reducing the energy barrier to proton hopping. Upon light irradiation, we can clearly observe an increase in measured current and gate response of the HPTSmodified mat (Figure 4). The extracted charge carrier mobility value of the sample upon light irradiation is around $7 \times 10^{-3} \mathrm{~cm}^{2} \mathrm{~V}^{-1} \mathrm{~S}^{-1}$, which is $40 \%$ larger than the value measured in the dark. This relatively mild increase in charge mobility suggests that the deprotonated photoacid contributes additional charge bearing Grotthuss hopping sites following ESPT, thus contributing to increased protonic mobility. By accounting for our measured conductivity values for before and after light irradiation, and while using the Nernst-Einstein relation, we find a large 4.5 folds increase in the extracted protonic charge carrier concentration, from $\sim 2 \times 10^{17} \mathrm{~cm}^{-3}$ at dark to $\sim 9 \times 10^{17} \mathrm{~cm}^{-3}$ under irradiation. This confirms that excitation with light adds charge carriers to the system resulting in an enhanced conductivity. This significant contribution is much greater than the 38\% increase reported by Gorodetsky and coworkers in drop-casted proton conductive films made with the cephalopod protein. ${ }^{[11,25-26]}$ This adds weight to our working hypothesis that covalent doping may be more effective than electrostatic binding for the augmentation of proteinbased materials. Our results of this section imply that dopants that are closer to the surface of the protein, meaning to the hydrogen-bond network, contribute significantly to the protonic transport than electrostatically bound counterparts, especially as the latter can result in binding within water protected binding sites, hence not participating in the hydrogen-bond network.

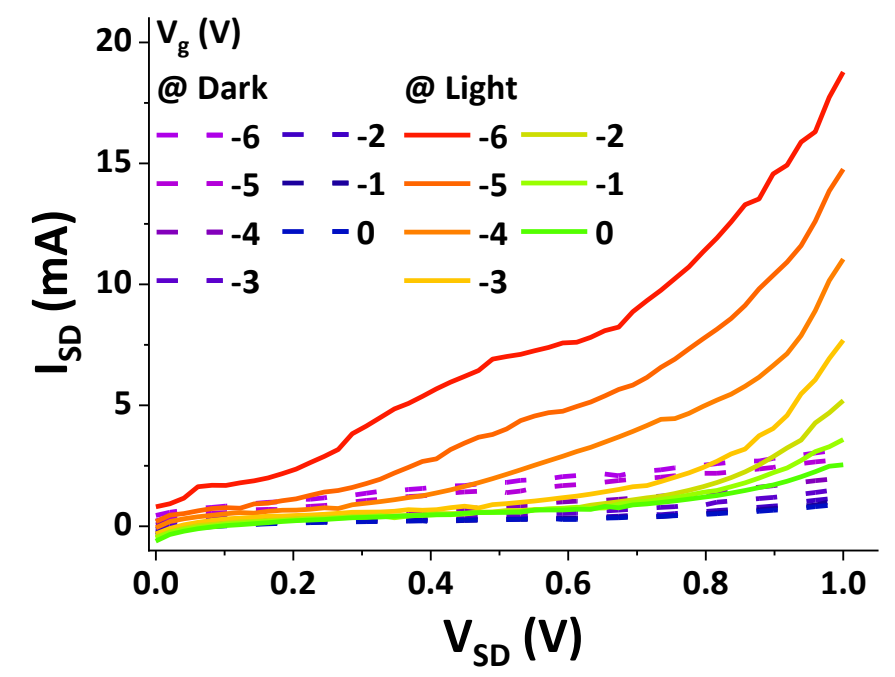

Figure 4. Transistor measurements of the photoacid-modified BSA mat. FET response across the 4 equivalents HPTS-tethered BSA mat at different gate voltages and upon light irradiation.

\section{Photobase-modified protein biopolymer}

In the second part of our study, we wish to explore the light-modulation response of a photobasemodified BSA mat, in which we chose here the 9-aminoacridine (9AA) photobase as the dopant 
molecule. Acridine is known for its photobasicity capable of ESPC, with a reported $\mathrm{p} K_{\mathrm{a}}$ of 5.1 and $\mathrm{p} K_{\mathrm{a}}{ }^{*}$ of $10.7,{ }^{[47]}$ meaning that it is relatively a weak photobase. Importantly, and the main reason for our choice here, acridine light absorption is around $390 \mathrm{~nm}$ with a tail extending into the visible, whereas the amino group redshifts this absorption to around $400 \mathrm{~nm}$. Furthermore, the amino groups render the molecule to be amenable to EDC coupling, pervasive in biological coupling to carboxylic acid (Figure 5a for the one-step conjugation schematic of 9AA to the BSA mat). Unlike the photoacid-modified mats scenario, we have two different hypotheses for the photobase-mats. As stated, the type of photobase that we use here undergoes ESPC, and accordingly, two very different outcomes can take place upon light irradiation (Figure 5b). i) The ESPC process of the photobase will result in a decrease in protonic charge carriers, and consequently, a decrease in the measured ionic conduction. ii) When water molecules serve as proton donors for the photobase, it will result in water deprotonation and the formation of hydroxide anions, ${ }^{[47-48]}$ hence an increase in a different charge carrier concentration, and consequently, an increase in the measured ionic conduction.

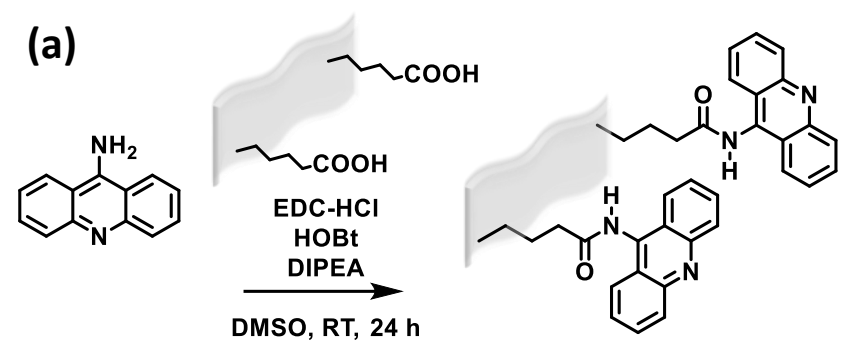

(b)
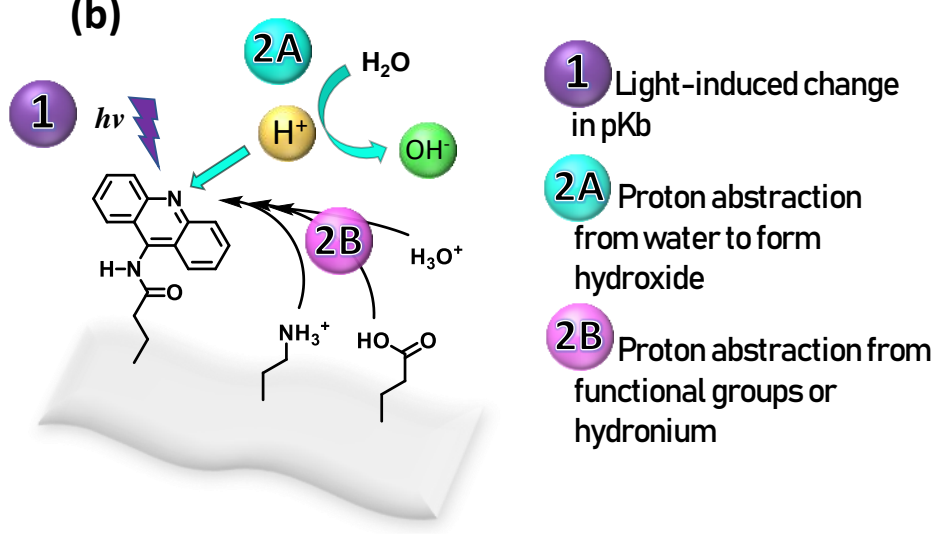

Figure 5. Photobase-modified BSA mat. (a) Scheme of 9AA modification and conjugation to the BSA mat. (b) Schematic of the light-induced processes within the 9AA-modified BSA mat.

As above, the first important step here is to validate the ESPC process of 9AA upon tethering to the BSA mat. For the photobase to undergo ESPC, there must be a proton donor (DH) nearby: $\mathrm{RN}+\mathrm{DH} \stackrel{h v}{\longrightarrow} \mathrm{RN}^{*}+\mathrm{DH} \underset{\text { deprot. }}{\stackrel{E S P C}{\longrightarrow}} \mathrm{RNH}^{+*}+\mathrm{D}^{-}$. As similar to photoacids, since the protonated and deprotonated species of the photobase have different emission wavelengths, it is straightforward to observe the ESPC process and its dynamicity using fluorescence measurements. The steady- 
state emission spectrum of the 9AA-modified BSA mat shows that indeed we can observe the $\mathrm{RNH}^{+*}$ species upon light irradiation, however, its intensity is lower compared to the one of the $\mathrm{RN}^{*}$ species (Figure 6a), which is probably due to a rather poor ESPC process, which requires proton abstraction from a donor, in contrast to the ESPT process. To decipher the rate constants of the ESPC process we turned to time-resolved measurements (Figure 6b). Similar to the $\mathrm{ROH}^{*}$ decay of the HPTS-BSA mat, also the transient decay of the $\mathrm{RN}^{*}$ species of the 9AA-BSA mat consists of a fast decay, attributed to the ESPC process, with an extracted lifetime of around 220 ps, hence, $k_{E S P C}=4.5 \times 10^{9} \mathrm{~s}^{-1}$. Unlike the immense body of work concerning the photophysical properties of the HPTS photoacid, there is not much knowledge on the photobasicity of 9AA. ${ }^{[49-50]}$ Very interestingly, we found here that the ESPC efficiency of the 9AA solvated in an aqueous solution is very poor with a lifetime of few ns's $\left(k_{E S P C} \sim 0.1 \times 10^{9} \mathrm{~s}^{-1}\right)$ (Figure S2), meaning that the covalent binding of 9AA to the BSA mat highly increases the photobasicity strength of 9AA, and accordingly the ESPC efficiency of this photobase. Figure $6 \mathrm{~b}$ also shows the slower decay of the $\mathrm{RNH}^{+*}$ peak.
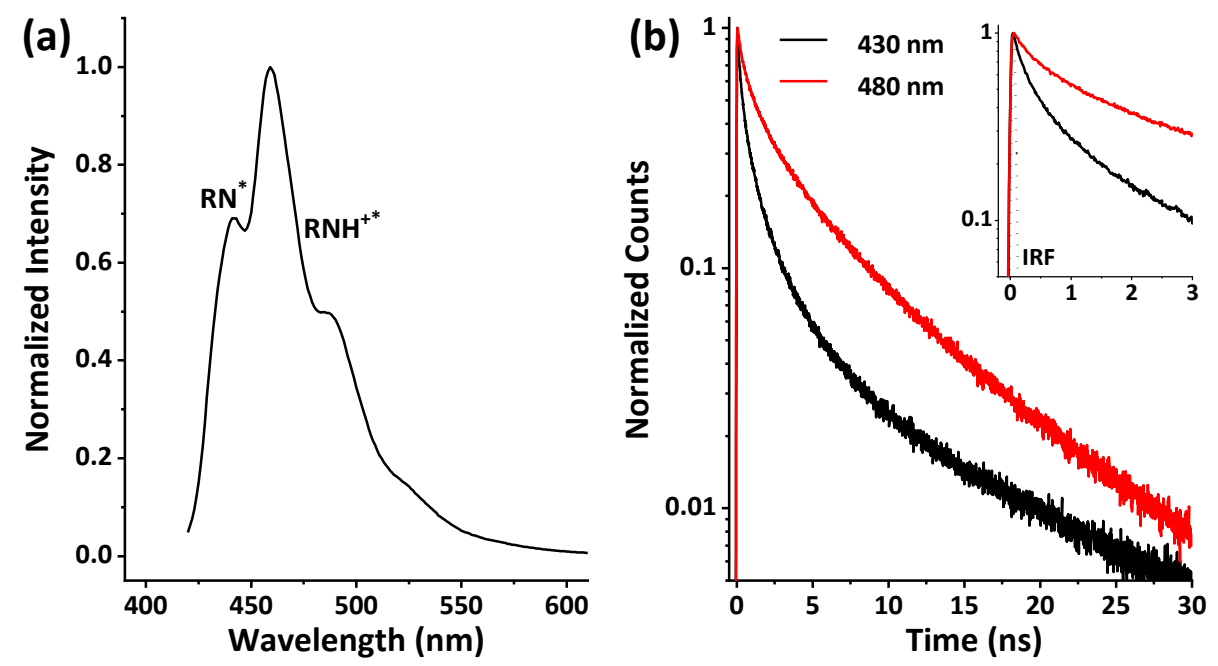

Figure 6. Excited-state dynamics of photobase-modified BSA mat. (a) Steady-state and (b) time-resolved fluorescence of the 9AA-tethered BSA mat, with $\lambda_{\mathrm{ex}}=390 \mathrm{~nm}$. The inset in (b) is a zoom-in of the first $3 \mathrm{~ns}$, emphasizing the difference between the decay of $\mathrm{RN}^{*}$ (at $430 \mathrm{~nm}$ ) to the one of the $\mathrm{RNH}^{+*}$ (at $480 \mathrm{~nm}$ ), along with the IRF.

After establishing that 9AA can undergo ESPC within the BSA mat with much greater efficiency compared to 9AA in solution, we turned to follow the light-modulated electrical response of the 9AA-tethered BSA mat. These measurements will allow us to decipher which one of our two hypotheses is taking place upon light irradiation, i.e., a reduction in protonic charge carriers manifested in lower conductivity or an increase in hydroxide charge carriers manifested in higher conductivity. First, we follow the change in measured bulk conductivity of the 9AA-BSA mat using AC impedance. Unlike the functionalization with HPTS, which introduced oxo-acids, and accordingly improved the hydrogen bonds network by adding proton hopping sites for the 
Grotthuss-like proton transport mechanism, the functionalization with 9AA shows an opposite effect. The measured protonic conductivity (in the dark) of the 9AA-BSA mat is lower compared with the native unmodified BSA mat, and with more equivalents of 9AA added, the less conductive the functionalized BSA mat (Figure $7 \mathrm{a}$ and $7 \mathrm{~b}$ and Table 1). The acridine derivative has a large hydrophobic aromatic surface with no oxo-acids, and this hydrophobic character of 9AA is anticipated to disrupt the H-bonds network, thus inhibiting proton transport in a Grotthuss-like mechanism. This result is in line with a previous study showing the effect of protein surface hydrophobicity in the measured protonic conductivity. ${ }^{[30]}$ Importantly, upon light-irradiation, we observed an increase in measured conductivity across the 9AA-tethered BSA mats (Figure 7a and $7 b$ ), hence validating one of our hypotheses, which is the light-induced formation of hydroxides (albeit in reductio ad absurdum, as there is no other explanation for the increase in bulk conductance upon light irradiation). Similar to the photoacid-modified mat, the more equivalents of 9AA within the mat, the stronger the light-response, reaching an impressive $3.6 \pm 0.5$ folds increase at added 4 equivalents of 9AA, with measured conductivity values of $0.04 \pm 0.004$ and $0.14 \pm 0.01 \mathrm{mS} \cdot \mathrm{cm}^{-1}$ in the dark and under irradiation with $405 \mathrm{~nm}$ laser, respectively (Table 1). As above, we can use the light as a gating source, and thereby manipulating the increase in ionic conductivity by the power of the light (Figure 7c).
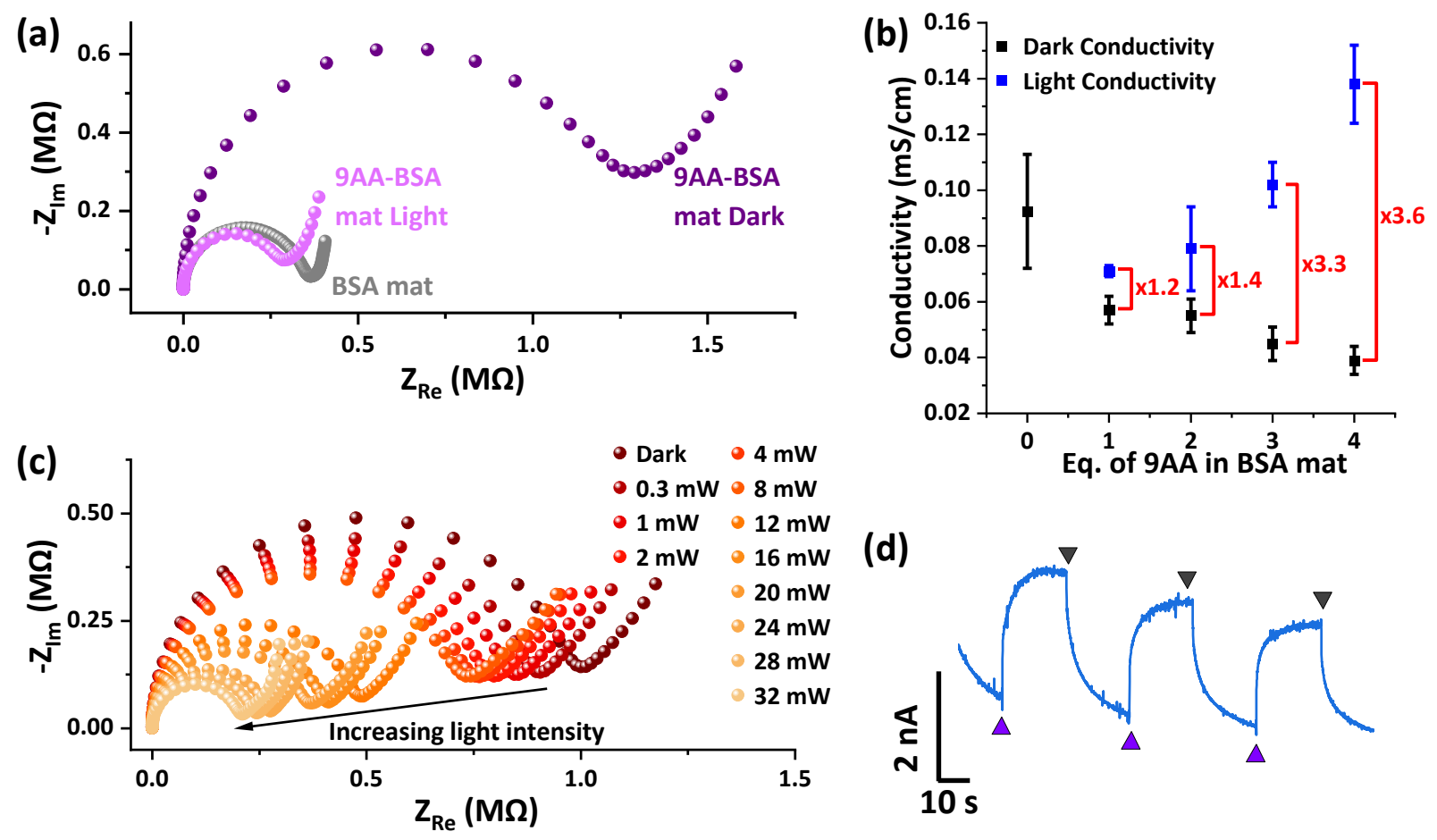

Figure 7. Light-modulated electrical response of photobase-modified BSA mat. (a) Impedance measurements of the BSA mat together with the 4 equivalents 9AA-tethered BSA mat at dark and under illumination. (b) A summary of the extracted conductivities in dark and light. (c) Light modulation of the impedance for the 4 equivalents 9AA-tethered BSA mat as a function of light intensity. (d) Current-time measurements of the 4 equivalents 9AA-tethered BSA upon intervals of light irradiation. 
We further used DC-bias I-T measurements to elucidate the dynamicity of the process. However, as discussed above, DC measurements using our electrodes for following hydroxide conductivity should be taken with a grain of salt. In accordance with our impedance measurements, also our transient I-T measurements show an increase in measured current upon light irradiation (Figure 7d). Like our HPTS-BSA mat measurements, the photo-modulation of the 9AA-BSA mat is also reversible with time-response on the second's timescales. However, the photocurrent magnitude of the 9AA mat pales in comparison to that of the HPTS mat (Figure S3), which can be ascribed to the described different role and reactivity of our gold electrodes. Unlike protons, hydroxides will rapidly accumulate on the surface of the electrodes, resulting in a rapid saturation of the measured I-T response, as observed in our measurements. In addition, due to the low photocurrent of the 9AA-tethered BSA mat, our I-T response is 'riding' the low-magnitude very rapid sharp increase in measured photocurrent ascribed to the photoelectric effect due to irradiation of the gold electrode, as can be observed in our control of the unmodified BSA mat (Figure S3). Overall, the 9AA-tethered BSA mat should have both proton transport capabilities as well as light-induced hydroxide transport capabilities. Accordingly, the mat should possess an ambipolar FET behavior under illumination. However, due to the discussed ion-blocking nature of our electrodes toward hydroxides, we could not observe the hydroxide mobility in our transistor measurements, and we observed only the protonic charge carriers FET response of the 9AA-BSA mat (Figure S4). ${ }^{[51]}$

\section{Conclusions}

In summary, we have developed here a light-modulated system based on BSA electrospun mats, whereas the ionic charge carrier's chemical nature, as well as the concentration of charge carriers, can be modulated with light using different functionalization of photoacids and photobases. We showed that both the photoacid (HPTS) and photobase (9AA) covalently-tethered BSA mats exhibit a large increase in measured ionic conductivity of 5.4 and 3.6 folds, respectively. However, we claim that this increase in ionic conductivity is a result of different processes. For the HPTStethered BSA mat, the conductivity increase is due to an increase in protonic charge carriers following the ESPT process of HPTS, whereas for the 9AA-tethered BSA mat is due to an increase in hydroxide concentration following the ESPC process of 9AA and deprotonation of water molecules. Importantly, our measured ESPT and ESPC efficiency of the HPTS- and 9AA-tethered BSA mats, respectively, are greater than the efficiency of the solvated molecule, indicating that the covalent connection to the surface of the protein fiber is essential for such improved efficiency. It also highlights the fundamental role of the protein surface in dictating the ionic charge transport across the material. Our new light-responsive biopolymers are highly attractive for numerous applications involving photoelectrochemical processes, such as in energy storage (e.g., batteries, capacitors), energy production (e.g., fuel cells), or ion pumping. The main advantage of using a biopolymer over a synthetic polymer pertains to the environmental, sustainable, and green 
chemistry nature of the biopolymer, as no synthesis is required for its formation, and the protein used here is essentially a recycle of one of the main wastes of the bovine industry. Due to the protein-based nature of the biopolymer, it is also biocompatible and biodegradable, hence promoting its use for biomedical applications.

\section{Methods}

Electrospun BSA Mat. 14\% (w/v) BSA (MP Biomedicals) was dissolved in 9:1 2,2,2Trifluoroethanol:Water, then $\beta$-mercaptoethanol $5 \%(\mathrm{v} / \mathrm{v})$ was added and allowed to react for 3 hours. The solution was injected using a flow controller over a custom rotating drum electrospinning collector system at a rate of $1.5 \mathrm{ml} /$ hour via $24 \mathrm{G}$ blunt tip about $10 \mathrm{~cm}$ above the collector. A bias of $12 \mathrm{kV}$ was applied to needle, with the drum collector grounded.

Synthesis of trisodium 8-acetoxypyrene-1,3,6-trisulfonic acid. Trisodium 8-hydroxypyrene1,3,6-trisulfonic acid $(0.400 \mathrm{~g}, 0.763 \mathrm{mmol})$ and sodium acetate $(0.006 \mathrm{~g}, 0.076 \mathrm{mmol})$ were suspended in acetic anhydride $(0.19 \mathrm{M}, 4.0 \mathrm{~mL})$ and subjected to microwave irradiation for $1.5 \mathrm{~h}$ at $300 \mathrm{~W}$ to a temperature of $130{ }^{\circ} \mathrm{C}$. After cooling to room temperature, the grey suspension was filtered, washed with acetone and dried under high vacuum to yield a grey powder $(0.418 \mathrm{~g}, 0.732$ mmol, 96\% yield). The product was confirmed with NMR (Figure S5).

Synthesis of 8-acetoxypyrene-1,3,6-trisulfonyl chloride. Trisodium 8-acetoxypyrene-1,3,6trisulfonic acid $(0.100 \mathrm{~g}, 0.177 \mathrm{mmol})$ was suspended in thionyl chloride $(0.18 \mathrm{M}, 1 \mathrm{~mL})$ with a catalytic amount of DMF (1 drop). The suspension was heated for 5 hours at $60^{\circ} \mathrm{C}$. After heating, the orange solution was poured into minimal ice water $(10 \mathrm{~mL})$ yielding an orange precipitate that was collected, washed with water and dried under high vacuum $(0.073 \mathrm{~g}, 0.131 \mathrm{mmol}, 74 \%$ yield). The product was confirmed with NMR (Figure S6).

Conjugation of 8-acetoxypyrene-1,3,6-trisulfonyl chloride to mat. 8-Acetoxypyrene-1,3,6trisulfonyl chloride $(5.3 \mathrm{mg}, 0.01 \mathrm{mmol}, 1 \mathrm{eq})$ was dissolved in DCM $(1 \mathrm{~mL})$ and triethylamine $(0.03 \mathrm{mmol}, 4.0 \mu \mathrm{L})$ added. A piece of BSA mat $(4.3 \mathrm{mg}, 64.5 \mathrm{nmol} \mathrm{BSA}=0.010 \mathrm{mmol}$ nucleophiles) was suspended in solution and agitated for 24 hours in the dark. After elapsed time, the mat was washed and solvent exchanged for 1 hour, in triplicate, with first DCM, then ethanol, then water. The final product is an orange-yellow colored mat that does not leach dopant in common solvents.

Conjugation of 9-aminoacridine to mat. 9-Aminoacridine (1.5 mg, $0.008 \mathrm{mmol}, 1 \mathrm{eq})$ and $\mathrm{N}$ hydroxybenzotriazole $(1.2 \mathrm{mg}, 0.008 \mathrm{mmol}, 1 \mathrm{eq})$ were added to a piece of BSA mat $(5.0 \mathrm{mg}, 75.3$ nmol $\mathrm{BSA}=0.008 \mathrm{mmol}$ electrophiles). A mix of 1-(3-Dimethylaminopropyl)-3ethylcarbodiimide hydrochloride $(1.5 \mathrm{mg}, 0.008 \mathrm{mmol}, 1 \mathrm{eq})$ and $\mathrm{N}, \mathrm{N}$-diisopropylethylamine $(2.7$ $\mu \mathrm{L}, 0.015 \mathrm{mmol}, 2 \mathrm{eq}$ ) were added to the suspension, total volume diluted to $0.5 \mathrm{~mL} \mathrm{DMSO}$, and agitated for 24 hours in the dark. After elapsed time, the mat was washed and solvent exchanged 
for 1 hour, in triplicate, with first DMSO and then water. The final product is a white-colored mat that does not leach dopant in common solvents.

Spectroscopy. Steady-state fluorescence measurements were taken with a Fluorolog-3 (Horiba) spectrofluorometer. The fluorescence lifetimes were measured by time-correlated single-photon counting (TCSPC) using a CHIMERA spectrometer (Light Conversion) with an excitation wavelength of $405 \mathrm{~nm}$. The laser system was based on a $10 \mathrm{~W}$ Nd-YAG-based laser amplifier (PHAROS, Light-Conversion) with pulses of <190 fs, operating at $1 \mathrm{MHz}$ (pulse energy of $10 \mu \mathrm{J}$ ). The laser beam (at $1030 \mathrm{~nm}$ ) is seeded into an optical parametric amplifier (ORPHEUS, LightConversion) followed by second and fourth harmonic generation. To calculate the lifetime, the fluorescence decay curves were analyzed with CarpetView fitting program.

Electrical Measurements. Electrochemical impedance spectroscopy (Biologic MTZ-35) was performed on $200 \mathrm{~nm}$ thick custom gold electrodes evaporated onto glass, $1 \mathrm{~mm}$ wide, and spaced $0.5 \mathrm{~mm}$ apart. The mat was laid on top of the electrodes, gently pressed with a glass slide to create good contact and remove excess moisture. Dark measurements were performed in a shaded environment. Light measurements were performed using a $35 \mathrm{~mW}\left(0.44 \mathrm{~W} / \mathrm{cm}^{2}\right)$ laser pen (JD850 ), positioned such that the entire area of the mat in contact and between electrodes is illuminated. Illumination began $20 \mathrm{~s}$ before the experiment was begun, then continued during the length of measure ( $40 \mathrm{~s})$. Current-Time and Transistor DC measurements (Keysight Technologies B2901A) were performed similarly, turning the light on and off at specified intervals by a chopper. Irradiation power was controlled by a neutral density filter. Gold finger electrodes distanced at $2.5 \mathrm{~mm}$ were used for the impedance measurements and current-time measurements. Interdigitated gold electrodes with $70 \mu \mathrm{m}$ distance were used for the transistor measurements.

\section{Acknowledgments}

S.M. thanks the PBC fellowship of Israel's council of higher education for financial support. N.A. thanks the Binational Science Foundation (grant number 2018239), the Ministry of Science and Technology (grant numbers 3-16243 and 3-16312), and the Volkswagen Foundation - Research Cooperation Lower Saxony - Israel (ZN 3625) for financial support. We thank the Russel Berrie Nanotechnology Institute (RBNI) and the Grand Technion Energy Program (GTEP) for their support in equipment use.

\section{Declaration of Interests}

The authors declare no competing interests.

\section{References}


[1] M. Forsyth, L. Porcarelli, X. E. Wang, N. Goujon, D. Mecerreyes, Acc. Chem. Res. 2019, $52,686$.

[2] Z. F. Wang, M. S. Zhu, Z. X. Pei, Q. Xue, H. F. Li, Y. Huang, C. Y. Zhi, Mater. Sci. Eng., $R$ 2020, 139.

[3] E. H. Majlan, D. Rohendi, W. R. W. Daud, T. Husaini, M. A. Haque, Renewable \& Sustainable Energy Reviews 2018, 89, 117.

[4] Z. F. Pan, L. An, T. S. Zhao, Z. K. Tang, Prog. Energy Combust. Sci. 2018, 66, 141.

[5] M. Rezakazemi, M. Sadrzadeh, T. Matsuura, Prog. Energy Combust. Sci. 2018, 66, 1.

[6] K. S. Ngai, S. Ramesh, K. Ramesh, J. C. Juan, Ionics 2016, 22, 1259.

[7] S. Gottesfeld, D. R. Dekel, M. Page, C. Bae, Y. Yan, P. Zelenay, Y. S. Kim, J. Power Sources 2018, 375, 170.

[8] W. White, C. D. Sanborn, R. S. Reiter, D. M. Fabian, S. Ardo, J. Am. Chem. Soc. 2017, 139, 11726.

[9] X. Xie, G. A. Crespo, G. Mistlberger, E. Bakker, Nat. Chem. 2014, 6, 202.

[10] S. Haghighat, S. Ostresh, J. M. Dawlaty, J. Phys. Chem. B 2016, 120, 1002.

[11] D. D. Ordinario, L. Phan, Y. Van Dyke, T. Nguyen, A. G. Smith, M. Nguyen, N. M. Mofid, M. K. Dao, A. A. Gorodetsky, Chem. Mater. 2016, 28, 3703.

[12] W. White, C. D. Sanborn, D. M. Fabian, S. Ardo, Joule 2018, 2, 94.

[13] H.-Q. Liang, Y. Guo, Y. Shi, X. Peng, B. Liang, B. Chen, Angew. Chem. Int. Edit. 2020, 59, 7732.

[14] A. M. Rice, C. R. Martin, V. A. Galitskiy, A. A. Berseneva, G. A. Leith, N. B. Shustova, Chem. Rev. 2020, 120, 8790.

[15] A. B. Kanj, A. Chandresh, A. Gerwien, S. Grosjean, S. Bräse, Y. Wang, H. Dube, L. Heinke, Chem. Sci. 2020, 11, 1404.

[16] S. Garg, H. Schwartz, M. Kozlowska, A. B. Kanj, K. Müller, W. Wenzel, U. Ruschewitz, L. Heinke, Angew. Chem. Int. Edit. 2019, 58, 1193.

[17] R. Ou, H. Zhang, V. X. Truong, L. Zhang, H. M. Hegab, L. Han, J. Hou, X. Zhang, A. Deletic, L. Jiang, G. P. Simon, H. Wang, Nat. Sustainability 2020, 3, 1052.

[18] S. S. Nagarkar, S. Horike, T. Itakura, B. Le Ouay, A. Demessence, M. Tsujimoto, S. Kitagawa, Angew. Chem. Int. Edit. 2017, 56, 4976.

[19] K. Müller, J. Helfferich, F. Zhao, R. Verma, A. B. Kanj, V. Meded, D. Bléger, W. Wenzel, L. Heinke, Adv. Mater. 2018, 30, 1706551.

[20] L. Wang, Q. Wen, P. Jia, M. Jia, D. Lu, X. Sun, L. Jiang, W. Guo, Adv. Mater. 2019, 31, 1903029.

[21] L. M. Tolbert, K. M. Solntsev, Acc. Chem. Res. 2002, 35, 19.

[22] B. Finkler, C. Spies, M. Vester, F. Walte, K. Omlor, I. Riemann, M. Zimmer, F. Stracke, M. Gerhards, G. Jung, Photochem. Photobiol. Sci. 2014, 13, 548.

[23] A. Yucknovsky, S. Mondal, A. Burnstine-Townley, M. Foqara, N. Amdursky, Nano Lett. 2019, 19, 3804.

[24] H. Peretz-Soroka, A. Pevzner, G. Davidi, V. Naddaka, M. Kwiat, D. Huppert, F. Patolsky, Nano Lett. 2015, 15, 4758.

[25] D. D. Ordinario, L. Phan, J.-M. Jocson, T. Nguyen, A. A. Gorodetsky, APL Mater. 2015, 3, 014907.

[26] D. D. Ordinario, L. Phan, W. G. Walkup Iv, J.-M. Jocson, E. Karshalev, N. Hüsken, A. A. Gorodetsky, Nat. Chem. 2014, 6, 596.

[27] G. Wu, P. Feng, X. Wan, L. Zhu, Y. Shi, Q. Wan, Sci. Rep. 2016, 6, 23578. 
[28] N. Amdursky, E. D. Głowacki, P. Meredith, Adv. Mater. 2019, 31, 1802221.

[29] N. Amdursky, X. Wang, P. Meredith, D. D. C. Bradley, M. M. Stevens, Adv. Mater. 2016, 28, 2692.

[30] S. Mondal, Y. Agam, R. Nandi, N. Amdursky, Chem. Sci. 2020, 11, 3547.

[31] C. Ma, J. Dong, M. Viviani, I. Tulini, N. Pontillo, S. Maity, Y. Zhou, W. H. Roos, K. Liu, A. Herrmann, G. Portale, Sci. Adv. 2020, 6, eabc0810.

[32] A. Pena-Francesch, H. Jung, M. A. Hickner, M. Tyagi, B. D. Allen, M. C. Demirel, Chem. Mater. 2018, 30, 898.

[33] Y. Agam, R. Nandi, T. Bulava, N. Amdursky, Materials Advances 2021, 2, 1739.

[34] N. Amdursky, M. M. Mazo, M. R. Thomas, E. J. Humphrey, J. L. Puetzer, J.-P. St-Pierre, S. C. Skaalure, R. M. Richardson, C. M. Terracciano, M. M. Stevens, J. Mater. Chem. B 2018, 6, 5604 .

[35] N. Amdursky, X. Wang, P. Meredith, D. J. Riley, D. J. Payne, D. D. C. Bradley, M. M. Stevens, Adv. Mater. 2017, 29, 1700810.

[36] N. Amdursky, Phys. Chem. Chem. Phys. 2015, 17, 32023.

[37] N. Amdursky, M. H. Rashid, M. M. Stevens, I. Yarovsky, Sci. Rep. 2017, 7, 6245.

[38] N. Amdursky, R. Simkovitch, D. Huppert, J. Phys. Chem. B 2014, 118, 13859.

[39] R. Nandi, A. Yucknovsky, M. M. Mazo, N. Amdursky, J. Mater. Chem. B 2020, 8, 6964.

[40] B. Cohen, C. Martin Alvarez, N. Alarcos Carmona, J. Angel Organero, A. Douhal, J. Phys. Chem. B 2011, 115, 7637.

[41] N. Amdursky, Y. Lin, N. Aho, G. Groenhof, Proc. Natl. Acad. Sci. 2019, 116, 2443.

[42] R. Simkovitch, D. Huppert, J. Phys. Chem. A 2015, 119, 1973.

[43] C. D. Sanborn, J. V. Chacko, M. Digman, S. Ardo, Chem 2019, 5, 1648.

[44] S. Mondal, Y. Agam, N. Amdursky, Small 2020, 16, 2005526.

[45] M. Amit, S. Roy, Y. Deng, E. Josberger, M. Rolandi, N. Ashkenasy, ACS Appl. Mater. Interfaces 2018, 10, 1933.

[46] N. Agmon, Chem. Phys. Lett. 1995, 244, 456.

[47] E. Pines, D. Huppert, M. Gutman, N. Nachliel, M. Fishman, J. Phys. Chem. 1986, 90, 6366.

[48] J. R. Hunt, J. M. Dawlaty, J. Phys. Chem. A 2018, 122, 7931.

[49] R. V. Pereira, A. P. G. Ferreira, M. H. Gehlen, J. Phys. Chem. A 2005, 109, 5978.

[50] C. A. Smith, H. C. Chang, W. S. Struve, G. J. Atwell, W. A. Denny, J. Phys. Chem. 1995, 99, 8927.

[51] We are aware of a previous study by the group of M. Rolandi using hydrogenated Pd electrodes for FET measurements targeting hydroxides as charge carriers (Deng et al., Sci. Rep. 2013, 3, 2481). In our study, we have tried to use hydrogenated Pd electrodes for the FET measurements, but similar response was observed with them as with the gold electrodes. 